\title{
Disentangling Diagnosis and Management of Fibromyalgia
}

\author{
Ji-Hyoun Kang, M.D., Ph.D., Sung-Eun Choi, M.D., Dong-Jin Park, M.D., Ph.D., Shin-Seok Lee, M.D., Ph.D. \\ Division of Rheumatology, Department of Internal Medicine, Chonnam National University Medical School and Hospital, Gwangju, Korea
}

Fibromyalgia (FM) is a chronic pain condition characterized by widespread pain accompanied by symptoms such as fatigue, sleep disturbance, cognitive dysfunction, and mood disorder. The pathophysiology of FM has been unclear, leading to inconsistent diagnosis and ineffective management. Several diagnostic criteria for FM have been proposed in recent years, including the revised 2016 American College of Rheumatology (ACR) criteria, the criteria of the ACTTION-American Pain Society Pain Taxonomy (AAPT) group, and the modified 2019 Fibromyalgia Assessment Status (FAS) criteria. Despite the appearance of newer criteria for FM diagnosis, the 2016 ACR criteria demonstrate the best performance. Many randomized controlled studies and systematic reviews have shown the therapeutic efficacies of pharmacological and non-pharmacological treatments of FM. Nevertheless, further research is needed to develop better treatment options. (J Rheum Dis 2022;29:4-13)

Key Words. Fibromyalgia, Diagnosis, Therapy

\section{INTRODUCTION}

Fibromyalgia (FM) is a chronic, complex disorder characterized by widespread musculoskeletal pain and various associated symptoms (e.g., fatigue, sleep fragmentation, depressed mood, and anxiety) [1]. The prevalence of FM is estimated to be $1 \% \sim 5 \%$ in the general population; it varies depending on the diagnostic criteria applied [2]. Despite the high prevalence of FM, diagnosis often requires more than 2 years; patients see an average of 3.7 different physicians during that time [3]. FM and its comorbidities negatively impact daily activities and healthrelated quality of life (HRQoL); they impose a major socioeconomic burden on healthcare systems and patients [4].

In 2016, the International Association for the Study of Pain (IASP) introduced the term "nociplastic pain"; this pain is mechanically distinct from the nociceptive and neuropathic types of pain [5]. Although the underlying pathophysiology of nociplastic pain is not fully understood, the decreased inhibition or amplification of pain signaling in the peripheral and central nervous systems is presumed to have a prominent role [6]. Nociplastic pain is frequently observed in FM patients. Recently, the IASP proposed a new classification system for chronic pain in the 11th International Classification of Diseases [7]. In this classification, chronic primary and secondary pain syndromes are dissociated; FM is classified as a chronic primary pain syndrome. This classification system should facilitate the application of uniform criteria to healthcare statistics, clinical trials, and publications. Furthermore, it could improve the understanding and application of mechanism-based clinical treatment.

There have been continuous efforts to improve the diagnostic accuracy of FM over the past few decades. Classification criteria were proposed by the American College of Rheumatology (ACR) in 1990 [8], followed by the symptom-based 2010/2011 ACR criteria [1,9] and revised 2016 ACR criteria [10]. Other groups have since introduced their own diagnostic criteria to simplify the diagnostic process during routine clinical practice. In terms of pharmacotherapy for FM, many randomized trials and

\footnotetext{
Received : November 8, 2021, Revised : November 26, 2021, Accepted : November 27, 2021

Corresponding to : Shin-Seok Lee (iD http://orcid.org/0000-0001-6810-7355

Division of Rheumatology, Department of Internal Medicine, Chonnam National University Medical School and Hospital, 42 Jebong-ro, Dong-gu, Gwangju 61469, Korea. E-mail : shinseok@chonnam.ac.kr
}

Copyright (C) 2022 by The Korean College of Rheumatology.

This is an Open Access article, which permits unrestricted non-commerical use, distribution, and reproduction in any medium, provided the original work is properly cited. 
meta-analyses have assessed the therapeutic efficacies of several drugs (e.g., pregabalin, duloxetine, and milnacipran) [11]. Non-pharmacological management is based on patient education, exercise, and cognitive behavioral therapy (CBT) [11]. In this review, we provide an up-todate perspective regarding the diagnosis and management of FM.

\section{DIAGNOSIS OF FM}

Over the past few decades, extensive efforts have been made to improve the diagnostic and screening criteria for FM. The ACR introduced a set of criteria to distinguish FM from other chronic pain diseases in 1990, as mentioned above (Table 1) [8]. The 1990 ACR criteria were based on tender point examinations, where at least 11 of 18 pain locations demonstrated tenderness in patients with chronic pain for a diagnosis of FM. However, the 1990 ACR criteria had several disadvantages. The performance of the tender point examination was difficult for primary care physicians; it also neglected important associated symptoms (e.g., fatigue, cognitive dysfunction, and sleep disturbance) $[12,13]$. Thus, the ACR proposed new FM criteria in 2010/2011 [1,9].

The 2010/2011 ACR criteria for FM introduced the con- cept of "widespread pain" and excluded the tender point examination (Tables 2 and 3). These criteria also included a systemic symptom-based assessment of fatigue, sleep problems, and cognitive and somatic disturbances. However, the application of these criteria can lead to misclassification because the widespread pain index (WPI) used to ascertain the number of pain locations does not consider the spatial distribution of those locations. Furthermore, there is no definition of generalized pain; it is therefore difficult to discriminate between FM and localized functional pain syndromes, including myofascial pain syndrome. Smythe [14] succinctly described the limitations of the 2010/2011 ACR criteria as follows: dilution, inconsistency, loss of specificity, and the inability to identify comorbid FM in patients with other diseases. To solve these problems, revised criteria were released by the ACR in 2016 [10].

In the revised 2016 ACR criteria, generalized pain (rather than widespread pain) in at least four of five distinct body regions is required for a diagnosis of FM (Table 4). Although the 2016 criteria permit the coexistence of other diseases, clinical diagnosis of FM remains difficult because of criteria complexity and the influence of various comorbidities. To reduce the time required for diagnosis and promote application in daily practice, the

Table 1. American College of Rheumatology 1990 criteria for the classification of fibromyalgia

1. History of chronic widespread pain

Definition. Pain is considered widespread when all of the following are present: pain in the left side of the body, pain in the right side of the body, pain above the waist, and pain below the waist. Additionally, axial skeletal pain (cervical spine or anterior chest or thoracic spine or low back) must be present. In this definition, shoulder and buttock pain is regarded as pain for each involved side. "Low back" pain is considered lower segment pain.

2. Pain in 11 of 18 tender point sites on digital palpation

Definition. Pain, on digital palpation, must be present in at least 11 of the following 18 sites:

Occiput: bilateral, at the suboccipital muscle insertions.

Low cervical: bilateral, at the anterior aspects of the intertransverse spaces at $\mathrm{C} 5 \sim \mathrm{C} 7$.

Trapezius: bilateral, at the midpoint of the upper border.

Supraspinatus: bilateral, at origins, above the scapula spine near the medial border.

Second rib: bilateral, at the second costochondral junctions, immediately lateral to the junctions on upper surfaces.

Lateral epicondyle: bilateral, $2 \mathrm{~cm}$ distal to the epicondyles.

Gluteal: bilateral, in upper outer quadrants of buttocks in anterior fold of muscle.

Greater trochanter: bilateral, posterior to the trochanteric prominence.

Knee: bilateral, at the medial fat pad proximal to the joint line.

Digital palpation should be performed with an approximate force of $4 \mathrm{~kg}$.

For a tender point to be considered "positive," the patient must state that palpation was painful. "Tender" is not considered "painful."

For classification purposes, patients are diagnosed with fibromyalgia if both criteria are satisfied. Widespread pain must have been present for at least 3 months. The presence of a second clinical disorder does not exclude the diagnosis of fibromyalgia. Adapted from the article of Wolfe et al. (Arthritis Rheum 1990;33:160-72) [8]. 
Table 2. 2010 American College of Rheumatology diagnostic criteria

\section{Criteria}

A patient satisfies diagnostic criteria for fibromyalgia if the following three conditions are met:

1. Widespread pain index (WPI) $\geq 7$ and symptom severity (SS) scale score $\geq 5$, or WPI $3 \sim 6$ and SS scale score $\geq 9$.

2. Symptoms have been present at a similar level for at least 3 months.

3. The patient does not have a disorder that would otherwise explain the pain.

Ascertainment

1. WPI: note the number of areas in which the patient has had pain over the past week. In how many areas has the patient had pain? Score will be between 0 and 19 .

Shoulder girdle, left; hip (buttock, trochanter), left; jaw, left; upper back;

Shoulder girdle, right; hip (buttock, trochanter), right; jaw, right; lower back;

Upper arm, left; upper leg, left; chest; neck;

Upper arm, right; upper leg, right; abdomen;

Lower arm, left; lower leg, left;

Lower arm, right; lower leg, right.

2. SS scale score:

Fatigue

Waking unrefreshed

Cognitive symptoms

For each of the three symptoms above, indicate the level of severity over the past week using the following scale:

$0=$ no problem

$1=$ slight or mild problems, generally mild or intermittent

$2=$ moderate, considerable problems, often present and/or at a moderate level

3 = severe: pervasive, continuous, life-disturbing problems

Considering somatic symptoms in general, indicate whether the patient has:*

$0=$ no symptoms

$1=$ few symptoms

$2=$ a moderate number of symptoms

$3=$ a large number of symptoms

The SS scale score is the sum of the severity of the three symptoms (fatigue, waking unrefreshed, and cognitive symptoms) plus the extent (severity) of somatic symptoms in general. The final score is between 0 and 12 .

*Somatic symptoms that might be considered: muscle pain, irritable bowel syndrome, fatigue/tiredness, cognitive or memory problems, muscle weakness, headache, pain/cramps in the abdomen, numbness/tingling, dizziness, insomnia, depression, constipation, pain in the upper abdomen, nausea, nervousness, chest pain, blurred vision, fever, diarrhea, dry mouth, itching, wheezing, Raynaud's phenomenon, hives/welts, ringing in ears, vomiting, heartburn, oral ulcers, loss of/change in taste, seizures, dry eyes, shortness of breath, loss of appetite, rash, sun sensitivity, hearing difficulties, easy bruising, hair loss, frequent urination, painful urination, and bladder spasms. Adapted from the article of Wolfe et al. (Arthritis Care Res [Hoboken] 2010;62:600-10) [1].

Analgesic, Anesthetic, and Addiction Clinical Trial Translations Innovations Opportunities and Networks (ACTTION)-American Pain Society Pain Taxonomy (AAPT) group devised their own criteria. These criteria were followed by the modified 2019 Fibromyalgia Assessment Status (FAS) criteria $[15,16]$. These systems were designed to reflect the current understanding of FM; they also facilitate easy diagnosis and follow-up.

The ACTTION public-private partnership between the US Food and Drug Administration and the American Pain Society led the AAPT group to develop "core diagnostic criteria" with clinical utility for discriminating FM from chronic pain disorders (Figure 1) [15]. The AAPT FM working group developed these criteria to facilitate the identification of FM patients via simplified diagnostic criteria. The concept of "multisite pain" was proposed as a substitute for chronic widespread pain; pain was required in at least six of nine sites to support a diagnosis of FM. Our previous study showed that multisite pain was a stricter criterion than the WPI in the ACR criteria [17]. Moreover, for simplicity, the AAPT criteria only focused on sleep disturbance and fatigue among the various symptoms associated with FM, such that patients with mild or fluctuating symptoms were not identified. Salaffi et al. [16] compared the performances of the 2011 and 2016 ACR criteria with the performance of the AAPT 
Table 3. 2011 American College of Rheumatology diagnostic criteria

\section{Criteria}

A patient satisfies the modified ACR 2010 fibromyalgia diagnostic criteria if the following three conditions are met:

1. Widespread pain index (WPI) $\geq 7$ and symptom severity score $\geq 5$ or WPI of $3 \sim 6$ and symptom severity score $\geq 9$.

2. Symptoms have been present at a similar level for at least 3 months.

3. The patient does not have a disorder that would otherwise sufficiently explain the pain.

Ascertainment

1. WPI: note the number of areas in which the patient has had pain over the past week. In how many areas has the patient had pain? Score will be between 0 and 19.

Shoulder girdle, left; hip (buttock, trochanter), left; jaw, left; upper back;

Shoulder girdle, right; hip (buttock, trochanter), right; jaw, right; lower back;

Upper arm, left; upper leg, left; chest; neck;

Upper arm, right; upper leg, right; abdomen;

Lower arm, left; lower leg, left;

Lower arm, right; lower leg, right.

2. Symptom Severity Score: fatigue; waking unrefreshed; cognitive symptoms.

For each of these three symptoms, indicate the level of severity over the past week using the following scale:

$0=$ no problem

$1=$ slight or mild problems; generally mild or intermittent

2 =moderate; considerable problems; often present and/or at a moderate level

3 = severe: pervasive, continuous, life-disturbing problems

The Symptom Severity Score is the sum of the severity of the three symptoms (fatigue, waking unrefreshed, and cognitive symptoms) plus the sum of the number of the following symptoms occurringduring the previous 6 months: headaches, pain or cramps in the lower abdomen, and depression (0 3). The final score is between 0 and 12 .

Adapted from the article of Wolfe et al. (J Rheumatol 2011;38:1113-22) [9].

criteria. The AAPT criteria performed worst in terms of sensitivity, specificity, and classification accuracy. When we compared all six sets of criteria (i.e., the 1990, 2010, 2011, and 2016 ACR criteria, as well as the FAS and AAPT criteria), the AAPT criteria had the lowest diagnostic accuracy [17]. Because simplicity is prioritized in the AAPT criteria over diagnostic accuracy, caution is necessary when using these criteria to diagnose patients with chronic pain.

The modified 2019 FAS criteria (the original version was published in 2009) are detailed in Figure 2 [16]. These criteria use a simplified rating of chronic widespread pain, where pain in 19 body regions is merely classified as present or absent, rather than rated on a four-point numerical scale. Similar to the AAPT criteria, the modified FAS criteria focus only on fatigue and sleep quality to simplify the diagnostic process. Thus, the limitations of the modified FAS criteria are similar to the limitations of the AAPT criteria. Our previous study found that, similar to the AAPT criteria, the modified FAS criteria had lower diagnostic accuracy than did the 1990, 2010, 2011, and 2016 ACR criteria; the 2016 ACR criteria showed the best performance [17]. Clauw strongly recommended that the 2011/2016 ACR criteria be used in clinical practice and research because they constitute a continuous, quantitative measure [18]. In summary, despite the development of newer criteria, the 2016 ACR criteria remain the most useful for clinical and epidemiological studies that require identification of FM patients.

\section{TREATMENT OF FM}

\section{Pharmacological management}

Several clinical practice guidelines [11,19-22] and meta-analyses [23-25] have been published to guide the pharmacological treatment of FM as shown in Table 5. However, only three drugs (pregabalin, duloxetine, and milnacipran) have been approved by the US Food and Drug Administration for the treatment of FM. Pregabalin exerts its effects through the $\alpha 2-\delta$ protein, an auxiliary subunit of voltage-dependent calcium channels. Pregabalin improved pain and sleep disturbances in six randomized controlled trials [26]. A meta-analysis demonstrated that pregabalin improves pain, fatigue, sleep, and HRQoL, but not depression: however, the effect sizes were small [23]. Two serotonin and noradrenaline reuptake inhibitors (duloxetine and milnacipran) were shown to decrease pain, improve clinical condition (as perceived by the pa- 
Table 4. 2016 revised American College of Rheumatology diagnostic criteria

Criteria

A patient satisfies the modified 2016 fibromyalgia criteria if the following three conditions are met:

1. Widespread pain index (WPI) $\geq 7$ and symptom severity scale (SSS) score $\geq 5$, or WPI of $4 \sim 6$ and SSS score $\geq 9$.

2. Generalized pain, defined as pain in at least four of five regions, must be present. Jaw, chest, and abdominal pain are excluded from the generalized pain definition.

3. Symptoms have been generally present for at least 3 months.

4. A diagnosis of fibromyalgia is valid irrespective of other diagnoses. A diagnosis of fibromyalgia does not exclude the presence of other clinically important illnesses.

Ascertainment

1. WPI: note the number of areas in which the patient has had pain over the past week. In how many areas has the patient had pain? Score will be between 0 and 19

Left upper region (Region 1)

Jaw, left

Shoulder girdle, left

Upper arm, left

Lower arm, left

Left lower region (Region 3)

Hip (buttock, trochanter), left

Upper leg, left

Lower leg, left

\author{
Right upper region (Region 2) \\ Jaw, right \\ Shoulder girdle, right \\ Upper arm, right \\ Lower arm, right \\ Right lower region (Region 4) \\ Hip (buttock, trochanter), right \\ Upper leg, right \\ Lower leg, right
}

Axial region (Region 5)

Neck

Upper back

Lower back

Chest

Abdomen

\title{
2. SSS score
}

\section{Fatigue}

Waking unrefreshed

Cognitive symptoms

For each of the three symptoms above, indicate the level of severity over the past week using the following scale:

$0=$ no problem

$1=$ slight or mild problems, generally mild or intermittent

$2=$ moderate, considerable problems, often present and/or at a moderate level

3 = severe: pervasive, continuous, life-disturbing problems

The SSS score is the sum of the severity scores of the three symptoms (fatigue, waking unrefreshed, and cognitive symptoms) (0 9), plus the sum $(0 \sim 3)$ of the number of the following symptoms the patient has been bothered by that occurred during the previous 6 months:

1. Headaches $(0 \sim 1)$

2. Pain or cramps in lower abdomen $(0 \sim 1)$

3. Depression $(0 \sim 1)$

The final symptom severity score is between 0 and 12

The fibromyalgia severity scale is the sum of the WPI and SSS

Adapted from the article of Wolfe et al. (Semin Arthritis Rheum 2016;46:319-29) [10].

tient), reduce fatigue, improve depressed mood, and enhance HRQoL. However, for both drugs, the effect sizes were small for the improvements in pain and clinical condition; they were marginal for improvements in fatigue, sleep, and HRQoL [25].

In addition to these US Food and Drug Administration- approved drugs, the European League Against Rheumatism (EULAR) tentatively recommends amitriptyline, cyclobenzaprine, and tramadol as pharmacological treatments for FM. Amitriptyline, a tricyclic antidepressant, reportedly decreased pain and fatigue, while improving sleep and HRQoL in FM patients. However, the effect 


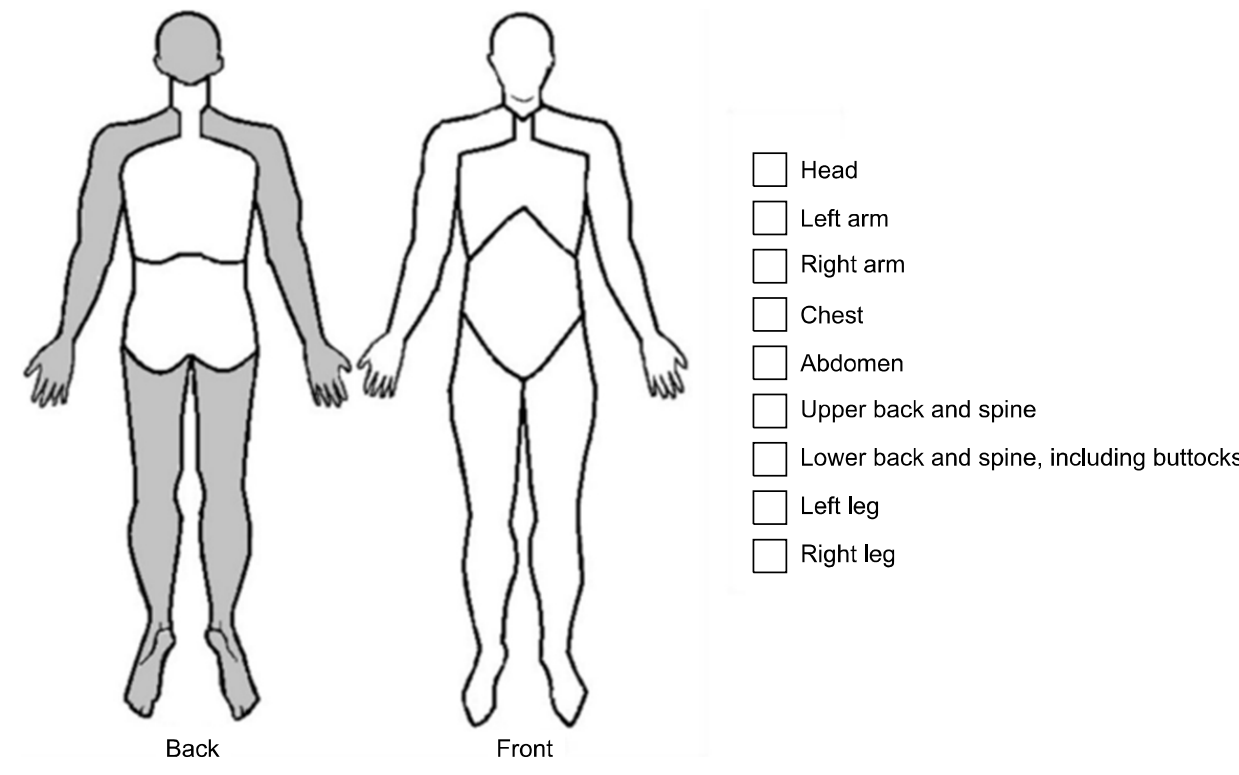

1. MSP is defined as pain at six or more sites among nine total sites 2. Moderate-to-severe sleep problems or fatigue

3. MSP plus fatigue or sleep problems must have been present for at least 3 months for a diagnosis of FM
Figure 1. AAPT diagnostic criteria for FM. AAPT: the ACTTIONAmerican Pain Society Pain Taxonomy (AAPT) group, MSP: multisite pain, FM: fibromyalgia. Adapted from the article of Arnold et al. (J Pain 2019;20: 611-28) [15].
Name and surname:

Please rate your level of fatigue:

No fatigue $\bigcirc \bigcirc \bigcirc \bigcirc \bigcirc \bigcirc \bigcirc \bigcirc \bigcirc \bigcirc \bigcirc$ Extreme fatigue Please rate the quality of your sleep:

Awoke well rested $\bigcirc \bigcirc \bigcirc \bigcirc \bigcirc \bigcirc \bigcirc \bigcirc \bigcirc \bigcirc \bigcirc$ Awoke very tired $\begin{array}{lllllllllll}0 & 1 & 2 & 3 & 4 & 5 & 6 & 7 & 8 & 9 & 10\end{array}$

Please indicate, in each of the body areas listed below, if you heave experienced pain and/or tenderness in the past week

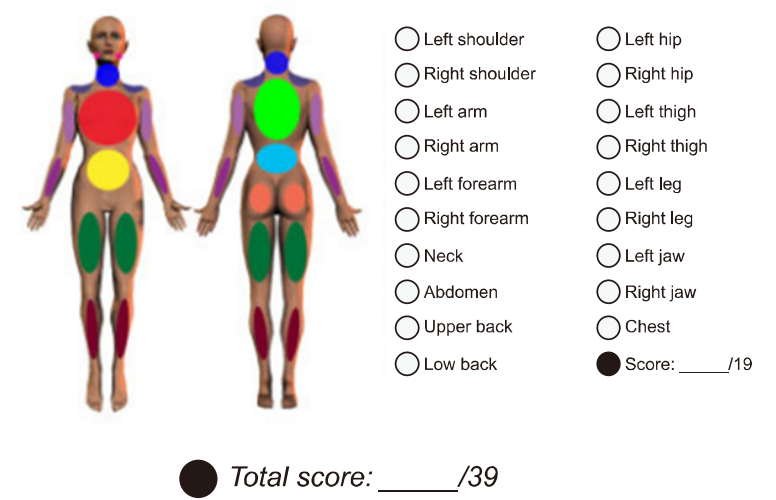

Figure 2. Modified 2019 FAS criteria for FM. FAS: fibromyalgia assessment status. Adapted from the article of Salaffi et al. (Rheumatology [Oxford] 2020;59:3042-9) [16].

sizes were small-to-moderate and its efficacy remains unconfirmed because of the methodological limitations of the corresponding clinical trials [27]. Cyclobenzaprine is a tricyclic antidepressant, currently licensed as a muscle relaxant. A meta-analysis of five trials showed that cyclobenzaprine improved global functioning in treated patients; it also elicited moderate improvements in pain and sleep quality [28]. Tramadol is an atypical analgesic that acts as a weak agonist of $\mu$-opioid receptors; it also acts as an inhibitor of serotonin and noradrenaline reuptake. When combined with acetaminophen, tramadol decreases pain and the Fibromyalgia Impact Questionnaire (FIQ) score; it also improves physical functioning [29]. The use of amitriptyline, cyclobenzaprine, and tramadol for the treatment of FM is also recommended by Canadian [21] and Italian practice guidelines [22].

Although the abovementioned drugs show efficacy for managing FM symptoms in randomized controlled trials and meta-analyses, the effect sizes are small and do not meet the threshold for a minimal clinically important difference. To overcome these limitations of monotherapy for FM, combinations of two or more drugs with different mechanisms of action have been attempted; additive or synergistic effects were expected to improve efficacy. The addition of milnacipran to pregabalin improved the pain, overall condition, and symptoms of FM patients who showed an incomplete response to pregabalin monotherapy in a randomized trial [30]. Similarly, patients who received pregabalin and duloxetine had better pain relief, physical function, and HRQOL outcomes than did patients who received either drug alone [31]. Thus, clini- 
Table 5. Comparison of the recommendations for pharmacological and non-pharmacological treatments

\begin{tabular}{|c|c|c|c|c|}
\hline & EULAR [11] & Canadian [21] & Italian [22] & German [19] \\
\hline \multicolumn{5}{|l|}{ Pharmacological management } \\
\hline Pregabalin & Weak for & Strong for & Strong for & - \\
\hline Duloxetine or milnacipran & Weak for & Strong for & Strong for & Weak for \\
\hline Amitriptyline & Weak for & Strong for & - & Weak for \\
\hline Cyclobenzaprine & Weak for & - & Strong for & Weak against \\
\hline Tramadol & Weak for & Strong against & - & - \\
\hline \multicolumn{5}{|l|}{ Non-pharmacological management } \\
\hline Exercise & Strong for & Strong for & Strong for & Strong for \\
\hline Cognitive behavioral therapy & Weak for & Strong for & Strong for & Strong for \\
\hline Physical therapies: acupuncture or hydrotherapy & Weak for & - & $\begin{array}{l}\text { Strong for } \\
\text { acupuncture only }\end{array}$ & - \\
\hline Meditative movement therapies: qigong, yoga or tai chi & Weak for & - & Strong for & Strong for \\
\hline Multicomponent therapies & Weak for & Strong for & - & Strong for \\
\hline
\end{tabular}

EULAR: European League Against Rheumatism.

cians should consider combination therapy for patients who show an inadequate response to any single therapeutic. However, combining different serotonin reuptake inhibitors (e.g., amitriptyline, fluoxetine, paroxetine, escitalopram, duloxetine, and milnacipran) should be performed cautiously to avoid the potentially lifethreatening condition known as serotonin syndrome.

Recently, the Spanish Society of Rheumatology issued guidelines that do not recommend any particular drug for FM, citing a lack of evidence. Instead, they provide information regarding drugs that are neither effective nor safe. They do not recommend non-steroidal anti-inflammatory drugs, opioids, or benzodiazepines because of insufficient evidence regarding their efficacy, as well as the risks of addiction or side effects. These drugs should not be prescribed during the initial stage of treatment; they should be stopped if already prescribed by another physician via consultation and shared decision-making with the patient.

\section{Non-pharmacological management}

Any treatment strategy for FM should incorporate non-pharmacological and pharmacological treatments in a comprehensive, multimodal approach. Physicians should encourage and support FM patients to participate in the non-pharmacological components of interventions as part of routine clinical practice. Clinical practice guidelines [11,21,22] and meta-analyses [24] recommend non-pharmacological approaches as first-line treatment for FM (Table 5).

Among the various non-pharmacological components of interventions, exercise is the only component strongly recommended by all guidelines [32]. Meta-analyses have shown that exercise improves pain, fatigue, sleep, depression, and FIQ scores. Aerobic, aquatic, resistance, and strengthening exercises are considered equally effective for relieving FM symptoms [33-35]. Patients are encouraged to begin at a level of intensity immediately below their current capacity; they should then gradually increase the duration and intensity until they are engaging in 20 30 minutes of low-to-moderate intensity exercise $2 \sim 3$ times per week [36]. The main challenge is maintenance of patient motivation; support from family, friends and physicians is important in this regard, both for beginning and adhering to long-term exercise regimens. CBT is recommended by most guidelines, although the strength of the recommendation varies [11,21,22]. Traditional and acceptance-based CBT are both effective for reducing pain, negative mood, and disability; they are also effective for improving HRQoL [37]. Similarly, internet-based CBT (ICBT) reduces negative mood and disability; it improves HRQoL [38]. However, pain reduction of at least $50 \%$ has not been demonstrated during the use of ICBT. Guided ICBT is generally superior to unguided interventions for reducing symptom severity and improving therapeutic outcomes in mental disorders. Thus, ICBT may be useful for FM patients with mild-tomoderate symptoms, while severe patients should be managed in a specialized setting through traditional and acceptance-based CBT [38]. When logistical considerations interfere with the provision of CBT, ICBT can be a good therapeutic option. 
Complementary and alternative therapies vary greatly in terms of both the quality of relevant clinical trials and the level of evidence. The EULAR guidelines are the first to tentatively recommend various non-conventional therapies, including acupuncture, hydrotherapy, meditative movement therapies (e.g., qigong, yoga, and tai chi), and mindfulness-based therapy [11]. Most guidelines are reluctant to recommend these therapies because of their ambiguous mechanisms of action, methodological flaws, and inabilities to elicit sustained responses [39]. There is a clear gap between patient enthusiasm for these therapies and the amount of scientific research regarding their efficacies; for acceptance as mainstream treatments, complementary and alternative therapies require additional well-designed, controlled studies of safety and long-term efficacy.

Non-invasive brain stimulation, such as transcranial direct current stimulation (tDCS) and repetitive transcranial magnetic stimulation (rTMS), has yielded promising results in the treatment of chronic pain conditions including FM by targeting the motor cortex or left dorsolateral prefrontal cortex [40]. Recent meta-analyses have shown that tDCS and rTMS are safe and effective for relieving pain and related symptoms in FM patients $[41,42]$, in contrast to the findings of an earlier metaanalysis [43]. In particular, tDCS is approved for treatment of FM in Canada and the European Union. Improvements in clinical trial protocols, conduct, and reporting are needed to verify the clinical significance of these techniques for FM patients.

Because FM is a complex, multidimensional disease that involves various pathophysiologies, a multicomponent approach involving pharmacological and non-pharmacological treatments is usually recommended [44]. Empirical evidence indicates the superiority of multicomponent therapies over individual therapies. According to a meta-analysis of nine randomized controlled trials, multicomponent therapy is effective for reducing key FM symptoms such as pain, fatigue, and depressed mood; it is also effective for improving self-efficacy and physical fitness [45]. However, because the effects of multicomponent therapy have short durations, extra effort is needed to ensure that the benefits persist after treatment. Education, booster sessions, and self-help groups can all enhance patient motivation to continue with therapy.

\section{CONCLUSION}

Although continuous efforts have been made to improve the accuracy of FM diagnosis, the 2016 ACR criteria remain the most accurate; thus, these criteria should be used in clinical practice and epidemiological studies. Despite advances in pharmacological and non-pharmacological treatments, there is considerable room for improvement in the treatment of FM. At present, the most effective strategy is to combine various treatment modalities to reduce symptoms and promote optimal functioning. Because FM is a heterogeneous disorder in terms of symptoms and severity, as well as the response to treatment [46], treatment plans should be individualized based on each patient's characteristics.

\section{CONFLICT OF INTEREST}

No potential conflict of interest relevant to this article was reported.

\section{AUTHOR CONTRIBUTIONS}

J.H.K., S.E.C., D.J.P., and S.S.L. conceived and planned this literature review. J.H.K. and S.S.L. reviewed the literature and wrote the manuscript.

\section{REFERENCES}

1. Wolfe F, Clauw DJ, Fitzcharles MA, Goldenberg DL, Katz RS, Mease P, et al. The American College of Rheumatology preliminary diagnostic criteria for fibromyalgia and measurement of symptom severity. Arthritis Care Res (Hoboken) 2010;62:600-10.

2. Jones GT, Atzeni F, Beasley M, Flüß E, Sarzi-Puttini P, Macfarlane GJ. The prevalence of fibromyalgia in the general population: a comparison of the American College of Rheumatology 1990, 2010, and modified 2010 classification criteria. Arthritis Rheumatol 2015;67:568-75.

3. Choy E, Perrot S, Leon T, Kaplan J, Petersel D, Ginovker A, et al. A patient survey of the impact of fibromyalgia and the journey to diagnosis. BMC Health Serv Res 2010;10:102.

4. Kim SK, Kim SH, Lee CK, Lee HS, Lee SH, Park YB, et al. Effect of fibromyalgia syndrome on the health-related quality of life and economic burden in Korea. Rheumatology (Oxford) 2013;52:311-20.

5. Kosek E, Cohen M, Baron R, Gebhart GF, Mico JA, Rice ASC, et al. Do we need a third mechanistic descriptor for chronic pain states? Pain 2016;157:1382-6.

6. Fitzcharles MA, Cohen SP, Clauw DJ, Littlejohn G, Usui C, Häuser W. Nociplastic pain: towards an understanding of prevalent pain conditions. Lancet 2021;397:2098-110. 
7. Treede RD, Rief W, Barke A, Aziz Q, Bennett MI, Benoliel R, et al. Chronic pain as a symptom or a disease: the IASP Classification of Chronic Pain for the International Classification of Diseases (ICD-11). Pain 2019;160:19-27.

8. Wolfe F, Smythe HA, Yunus MB, Bennett RM, Bombardier C, Goldenberg DL, et al. The American College of Rheumatology 1990 criteria for the classification of fibromyalgia. Report of the multicenter criteria committee. Arthritis Rheum 1990;33:160-72.

9. Wolfe F, Clauw DJ, Fitzcharles MA, Goldenberg DL, Häuser W, Katz RS, et al. Fibromyalgia criteria and severity scales for clinical and epidemiological studies: a modification of the ACR Preliminary Diagnostic Criteria for Fibromyalgia. J Rheumatol 2011;38:1113-22.

10. Wolfe F, Clauw DJ, Fitzcharles MA, Goldenberg DL, Häuser W, Katz RL, et al. 2016 Revisions to the 2010/2011 fibromyalgia diagnostic criteria. Semin Arthritis Rheum 2016;46:319-29.

11. Macfarlane GJ, Kronisch C, Dean LE, Atzeni F, Häuser W, Fluß E, et al. EULAR revised recommendations for the management of fibromyalgia. Ann Rheum Dis 2017;76:318-28.

12. Choy EH, Arnold LM, Clauw DJ, Crofford LJ, Glass JM, Simon LS, et al. Content and criterion validity of the preliminary core dataset for clinical trials in fibromyalgia syndrome. J Rheumatol 2009;36:2330-4.

13. Fitzcharles MA, Boulos P. Inaccuracy in the diagnosis of fibromyalgia syndrome: analysis of referrals. Rheumatology (Oxford) 2003;42:263-7.

14. Smythe HA. Unhelpful criteria sets for "diagnosis" and "assessment of severity" of fibromyalgia. J Rheumatol 2011; 38:975-8.

15. Arnold LM, Bennett RM, Crofford LJ, Dean LE, Clauw DJ, Goldenberg DL, et al. AAPT diagnostic criteria for fibromyalgia. J Pain 2019;20:611-28.

16. Salaffi F, Di Carlo M, Farah S, Atzeni F, Buskila D, Ablin JN, et al. Diagnosis of fibromyalgia: comparison of the 2011/2016 ACR and AAPT criteria and validation of the modified Fibromyalgia Assessment Status. Rheumatology (Oxford) 2020;59:3042-9.

17. Kang JH, Choi SE, Xu H, Park DJ, Lee JK, Lee SS. Comparison of the AAPT fibromyalgia diagnostic criteria and modified FAS criteria with existing ACR criteria for fibromyalgia in Korean patients. Rheumatol Ther 2021;8: 1003-14.

18. Clauw D. Time to stop the fibromyalgia criteria wars and refocus on identifying and treating individuals with this type of pain earlier in their illness. Arthritis Care Res (Hoboken) 2021;73:613-6.

19. Sommer C, Alten R, Bär KJ, Bernateck M, Brückle W, Friedel $\mathrm{E}$, et al. [Drug therapy of fibromyalgia syndrome: updated guidelines 2017 and overview of systematic review articles]. Schmerz 2017;31:274-84. German.

20. Rivera Redondo J, Díaz Del Campo Fontecha P, Alegre de Miquel C, Almirall Bernabé M, Casanueva Fernández B, Castillo Ojeda C, et al. Recommendations by the Spanish Society of Rheumatology on fibromyalgia. Part 1: diagnosis and treatment. Reumatol Clin (Engl Ed) 2021 Oct 11 [Epub]. DOI:10.1016/j.reumae.2021.02.002.

21. Fitzcharles MA, Ste-Marie PA, Goldenberg DL, Pereira JX, Abbey S, Choinière M, et al. 2012 Canadian Guidelines for the diagnosis and management of fibromyalgia syndrome: executive summary. Pain Res Manag 2013;18:119-26.

22. Ariani A, Bazzichi L, Sarzi-Puttini P, Salaffi F, Manara M, Prevete I, et al. The Italian Society for Rheumatology clinical practice guidelines for the diagnosis and management of fibromyalgia Best practices based on current scientific evidence. Reumatismo 2021;73:89-105.

23. Derry S, Cording M, Wiffen PJ, Law S, Phillips T, Moore RA. Pregabalin for pain in fibromyalgia in adults. Cochrane Database Syst Rev 2016;9:CD011790.

24. Mascarenhas RO, Souza MB, Oliveira MX, Lacerda AC, Mendonça VA, Henschke N, et al. Association of therapies with reduced pain and improved quality of life in patients with fibromyalgia: a systematic review and meta-analysis. JAMA Intern Med 2021;181:104-12.

25. Welsch P, Üçeyler N, Klose P, Walitt B, Häuser W. Serotonin and noradrenaline reuptake inhibitors (SNRIs) for fibromyalgia. Cochrane Database Syst Rev 2018;2: CD010292.

26. Straube S, Derry S, Moore RA, McQuay HJ. Pregabalin in fibromyalgia: meta-analysis of efficacy and safety from company clinical trial reports. Rheumatology (Oxford) 2010;49: 706-15.

27. Häuser W, Petzke F, Üçeyler N, Sommer C. Comparative efficacy and acceptability of amitriptyline, duloxetine and milnacipran in fibromyalgia syndrome: a systematic review with meta-analysis. Rheumatology (Oxford) 2011;50: 532-43.

28. Tofferi JK, Jackson JL, O'Malley PG. Treatment of fibromyalgia with cyclobenzaprine: a meta-analysis. Arthritis Rheum 2004;51:9-13.

29. Bennett RM, Kamin M, Karim R, Rosenthal N. Tramadol and acetaminophen combination tablets in the treatment of fibromyalgia pain: a double-blind, randomized, placebo-controlled study. Am J Med 2003;114:537-45.

30. Mease PJ, Farmer MV, Palmer RH, Gendreau RM, Trugman $\mathrm{JM}$, Wang Y. Milnacipran combined with pregabalin in fibromyalgia: a randomized, open-label study evaluating the safety and efficacy of adding milnacipran in patients with incomplete response to pregabalin. Ther Adv Musculoskelet Dis 2013;5:113-26.

31. Gilron I, Chaparro LE, Tu D, Holden RR, Milev R, Towheed $\mathrm{T}$, et al. Combination of pregabalin with duloxetine for fibromyalgia: a randomized controlled trial. Pain 2016;157: 1532-40.

32. Thieme K, Mathys M, Turk DC. Evidenced-based guidelines on the treatment of fibromyalgia patients: are they consistent and if not, why not? Have effective psychological treatments been overlooked? J Pain 2017;18:747-56.

33. Bidonde J, Busch AJ, Schachter CL, Overend TJ, Kim SY, Góes SM, et al. Aerobic exercise training for adults with fibromyalgia. Cochrane Database Syst Rev 2017;6:CD012700.

34. Bidonde J, Busch AJ, Webber SC, Schachter CL, Danyliw A, Overend TJ, et al. Aquatic exercise training for fibromyalgia. Cochrane Database Syst Rev 2014;(10):CD011336.

35. Häuser W, Klose P, Langhorst J, Moradi B, Steinbach M, Schiltenwolf M, et al. Efficacy of different types of aerobic exercise in fibromyalgia syndrome: a systematic review and meta-analysis of randomised controlled trials. Arthritis Res Ther 2010;12:R79.

36. Busch AJ, Barber KA, Overend TJ, Peloso PM, Schachter CL. Exercise for treating fibromyalgia syndrome. Cochrane 
Database Syst Rev 2007;(4):CD003786.

37. Bernardy K, Klose P, Welsch P, Häuser W. Efficacy, acceptability and safety of cognitive behavioural therapies in fibromyalgia syndrome - a systematic review and meta-analysis of randomized controlled trials. Eur J Pain 2018;22: 242-60.

38. Bernardy K, Klose P, Welsch P, Häuser W. Efficacy, acceptability and safety of Internet-delivered psychological therapies for fibromyalgia syndrome: a systematic review and meta-analysis of randomized controlled trials. Eur J Pain 2019;23:3-14.

39. Perry R, Leach V, Davies P, Penfold C, Ness A, Churchill R. An overview of systematic reviews of complementary and alternative therapies for fibromyalgia using both AMSTAR and ROBIS as quality assessment tools. Syst Rev 2017;6:97.

40. Meeker TJ, Jupudi R, Lenz FA, Greenspan JD. New developments in non-invasive brain stimulation in chronic pain. Curr Phys Med Rehabil Rep 2020;8:280-92.

41. Lloyd DM, Wittkopf PG, Arendsen LJ, Jones AKP. Is transcranial direct current stimulation (tDCS) effective for the treatment of pain in fibromyalgia? A systematic review and meta-analysis. J Pain 2020;21:1085-100.

42. Su YC, Guo YH, Hsieh PC, Lin YC. Efficacy of repetitive transcranial magnetic stimulation in fibromyalgia: a systematic review and meta-analysis of randomized controlled trials. J Clin Med 2021;10:4669.

43. O'Connell NE, Marston L, Spencer S, DeSouza LH, Wand BM. Non-invasive brain stimulation techniques for chronic pain. Cochrane Database Syst Rev 2018;4:CD008208.

44. Nüesch E, Häuser W, Bernardy K, Barth J, Jüni P. Comparative efficacy of pharmacological and non-pharmacological interventions in fibromyalgia syndrome: network meta-analysis. Ann Rheum Dis 2013;72:955-62.

45. Häuser W, Bernardy K, Arnold B, Offenbächer M, Schiltenwolf M. Efficacy of multicomponent treatment in fibromyalgia syndrome: a meta-analysis of randomized controlled clinical trials. Arthritis Rheum 2009;61:216-24.

46. Yim YR, Lee KE, Park DJ, Kim SH, Nah SS, Lee JH, et al. Identifying fibromyalgia subgroups using cluster analysis: relationships with clinical variables. Eur J Pain 2017;21: 374-84. 